\title{
CLASES, COHORTES, PARTIDOS Y ELECCIONES Un análisis de la experiencia española (1986-1996)
}

\author{
JUAN JESÚS GONZÁLEZ RODRÍGUEZ \\ Sociología II. UNED
}

\section{PALABRAS CLAVE ADICIONALES}

Clase social, Análisis electoral, Partidos políticos, España.

\author{
ADDITIONAL KEYWORDS \\ Social Class, Political Parties, Elections, \\ Cleavages, Spain.
}

RESUMEN. El artículo tiene tres partes. En la primera se hace una presentación de algunas de las explicaciones más habituales sobre la evolución electoral durante la etapa socialista en España (más concretamente, la década 1986-96), tanto del lado de la sociología como de la politología. En la segunda se hace una interpretación de dicha evolución, poniendo el énfasis en el papel de las políticas de bienestar y en el conflicto entre gobierno y sindicatos, entendido como un conflicto acerca de dichas políticas. En la tercera se formulan, a partir de esta experiencia, algunas enseñanzas de utilidad para sociólogos y politólogos a la hora de explicar este tipo de procesos.

SUMMARY. This article is made up of three parts. The first one introduces to some of the most common explanations of electoral evolution during the Spanish Socialist period (specifically, the decade 1986-1996), from both the Sociology and the Political Science. The second one proposes an interpretation of this evolution, by way of emphasizing the role of Welfare policies, and the conflict between government and unions linked to these policies. The third one points out some of the lessons that both sociologists and political scientists may learn from this experience.

\footnotetext{
* En su primera versión, este artículo se presentó como ponencia en la sesión conjunta que con el título de "Clase, política y ciudadanía" celebraron los Grupos de Trabajo de Desigualdad y de Sociología Política en el Congreso Español de Sociología (A Coruña, 25 de septiembre de 1998).

E-mail: jgonzalez@poli.uned.es
}

\section{Revista Internacional de Sociología (RIS)}

Tercera Época, n 29 , Mayo-Agosto, 2001, pp. 91-113. 
RIS

REVISTA INTERNACIONAL DE SOCIOLOGIA

\section{INTRODUCCIÓN}

El propósito de este artículo es elaborar una explicación de la evolución electoral española desde 1986 a 1996. Al decir una explicación doy por supuesto que no excluye otras explicaciones alternativas, sino que puede ser complementaria de ellas. Comenzaré discutiendo algunas de las explicaciones más habituales, a fin de acotar los límites del debate. Presentaré, a continuación, mi propia interpretación del proceso político-electoral a lo largo de la década de referencia. Y terminaré formulando algunas de las enseñanzas que, a mi juicio, podemos extraer de él.

Una interpretación habitual en los años ochenta sostenía que el sistema de partidos que surge de la transición no tenía unas bases nítidas de carácter estructural y que la clase social nunca fue un factor determinante en la configuración del mapa electoral, de tal forma que factores relativos a la ideología, al liderazgo o al conflicto centro-periferia habían desplazado a los factores estructurales en dicha configuración.

Esta interpretación casaba bien con la idea de que, en ausencia de una sociedad civil suficientemente madura y articulada (herencia del antiguo régimen), las élites dirigentes disponen de un amplio margen de decisión política, con lo que las estrategias de los partidos se hacen relativamente independientes respecto a las líneas tradicionales de división social ligadas a la clase. Tales argumentos desembocan en alguna forma de desalineamiento respecto de lo que durante mucho tiempo se consideró el orden político genuino de la democracia industrial, entendido como "lucha democrática de clases", con lo que España pasaría así a engrosar la lista de países en que las bases tradicionales de la competición partidista se habrían descongelado.

Desde esta perspectiva, la experiencia de la transición habría tenido, entre otros, el efecto benéfico de contener los conflictos distributivos en favor de las grandes cuestiones de interés nacional: consolidación democrática, reorganización del Estado, superación de la crisis económica, etc. Y, pues no hay bien que por mal no venga, los problemas de la transición nos habrían transportado, por atajos imprevistos, a resultados más o menos próximos a la posmodernidad, con lo que, una vez más, el caso español se convertía en un caso llamativo de país capaz de llegar a un estadio pos (ya fuese posindustrial, posclasista o posmaterialista) sin necesidad de estaciones intermedias.

Puede, en efecto, que el papel predominante del PSOE durante la década 1982-1992, al desplegar una estrategia típica de partido omnivoro con su retórica de modernización nacional, contribuyese durante algún tiempo a mantener el espejismo de un orden posclasista. Pero lo cierto es que, tan pronto como el primer gobierno socialista puso en vías de solución las grandes cuestiones de interés nacional, incluida la integración europea, los conflictos distributivos (que habían estado reprimidos o latentes desde los Pactos de la Moncloa) 
volvieron al primer plano de la agenda política de mediados de los ochenta, dando por cerrado el ciclo de concertación social que se había iniciado a finales de los setenta.

En cuanto los españoles se sintieron liberados de las preocupaciones de la transición (riesgo de involución, grave crisis económica, etc.), volvieron a radicalizar sus demandas, aunque fuera de manera distinta de como lo habían hecho al principio de la transición, al desplazar la presión desde el ámbito de la distribución (salario directo) al de la re-distribución (salario social en cualquiera de sus formas: pensiones, reivindicaciones estudiantiles, etc.). Al mismo tiempo la nueva situación de estabilidad política y de recuperación económica daba por concluida la atención preferente que el partido socialista había prestado a las cuestiones de interés nacional en la primera legislatura y le emplazaba a desarrollar las reformas de corte socialdemócrata contenidas en su programa..

De hecho, las elecciones de 1986 fueron la última oportunidad del PSOE para ensayar una estrategia típica de partido omnivoro, con una campaña dominada por vagas promesas de modernización, tal como sugería el eslogan elegido para la ocasión: "Por buen camino". Esa fue la primera y última vez que las cuestiones económicas no estuvieron en el centro de la campaña socialista (incluso en 1982 lo habían estado, con la promesa, tan inoportuna, de 800 mil puestos de trabajo).

Por contraste, el escenario electoral de 1989 estuvo marcado por la extraordinaria presión sindical que llevó a la huelga general de diciembre de 1988 y que culminó en las negociaciones posteriores, cuando los sindicatos exigieron al gobierno que saldara la llamada deuda social, lo que dio lugar a una operación redistributiva sin precedentes y de consecuencias imprevisibles en aquel momento.

En este punto, las interpretaciones del proceso político-electoral tienden a bifurcarse, estando, por un lado, quienes defienden la capacidad de determinación de los factores estructurales, al modo marxista tradicional: los intereses de clase explican el comportamiento electoral, al tiempo que la demanda política, entendida en términos de clases con intereses específicos y contrapuestos, explica la oferta de los partidos (sus políticas). En nuestro caso, la estructura de clase de la España de los ochenta permite determinadas coaliciones electorales, las cuales dan lugar a la hegemonía política, pero sin que los partidos puedan modificar sustancialmente las posibilidades de coalición y, por ende, sus opciones de hegemonía hasta tanto se produzcan cambios estructurales (Rodríguez Menés, 1997).

Y están, por otro lado, quienes defienden la capacidad de los partidos y, sobre todo, de las élites para conformar la demanda política con arreglo a sus propias estrategias. En nuestro caso, el deslizamiento estratégico del PSOE hacia una operación redistributiva de gran calado, a raíz de la huelga general de 1988, sería tan sólo un buen ejemplo de la receptividad de los partidos omnívoros a las 
demandas del entorno (Torcal y Chhibber, 1995: 21), pues lo que mejor explica este tipo de procesos, especialmente en sociedades poco articuladas como la española, es la capacidad de los dirigentes políticos para moldear e integrar las reivindicaciones del entorno social. El razonamiento de estos autores es simétrico del anterior, siendo ahora la oferta la que nos explica la demanda, toda vez que las élites imprimen su propia racionalidad en la elaboración de la agenda política.

En ambos casos, opera alguna forma de reduccionismo. En el primero los intereses de clase se imponen como criterio de decisión electoral a costa de los partidos, convertidos en una especie de variable dependiente cuya acción política queda excluida del escenario. En la argumentación de Rodríguez Menés, la acción de los partidos sólo aparece como capacidad clientelista de los gobiernos, a modo de hipótesis ad hoc para explicar las anomalías en la formăción de coaliciones (la anomalía, en este caso, radica en el apoyo que la pequeña burguesía presta al PSOE en ciertas regiones; op cit: 101).

El comportamiento electoral se intenta explicar así desde el sociologismo más crudo, de tal suerte que la eventual modificación de una determinada coalición electoral (y de su correlato en términos de hegemonía política) depende no de lo que hagan los partidos, sino de factores tales como "la extensión y riqueza de la sociedad civil, (o) la complejidad, número e interacción entre criterios alternativos de diferenciación social" (op cit: 87).

Por su parte, el segundo reduccionismo razona a la inversa, como si los partidos operasen sobre una especie de tabula rasa fácilmente acomodable a sus estrategias. En principio, el modelo teórico que nos proponen Torcal y Chhibber parece apartarse de esta manera de razonar. Podemos resumirlo en un doble supuesto:

1. “...Las estrategias políticas adoptadas por las élites (...) llevan a la formación de vínculos entre los apoyos electorales otorgados a los partidos y las divisiones existentes en la sociedad (lo que) podría constituirse en el primer paso hacia la institucionalización de las bases sociales del sistema de partidos" (op cit: 11).

2. En sociedades poco articuladas como la española, los partidos políticos operan en una especie de "vacío organizacional", por lo que "las élites políticas disponen de una mayor libertad para modelar las preferencias de los votantes (...) Ello no significa que los políticos dispongan de libertad total a la hora de tomar posiciones (pero) dentro de un margen razonable, los líderes pueden influir de manera importante resaltando ciertos problemas y conflictos en lugar de otros" (op cit: 11).

Pero la interpretación final del proceso vuelve a incurrir en un problema de sobreestimación de la capacidad estratégica de las élites, en virtud de la cual la emergencia de la clase social como variable explicativa del voto socialista obedecería "a las estrategias políticas adoptadas por los líderes del PSOE y del 
PP, y a las políticas económicas y fiscales adoptadas por el gobierno socialista desde 1989" (op cit: 18), las cuales, a su vez, no serían, como ya vimos, más que un buen ejemplo de la receptividad de que hacen gala los partidos omnivoros ante las demandas del entorno social (en este caso, de los sindicatos).

El problema es que esta especie de plasticidad estratégica de los partidos está sujeta a constricciones precisas y que, en nuestro caso, el deslizamiento del PSOE hacia una política redistributiva (el llamado giro social) duró exactamente lo que permitió el ciclo económico. Porque si bien es verdad que el PSOE repitió su éxito electoral en 1989 (lo que ha sido interpretado como un reforzamiento del gobierno socialista ante la "escasa repercusión política del descontento social", tal como han hecho recientemente Fusi y Palafox (1997: 396), este tipo de interpretaciones pasa por alto el verdadero significado del deslizamiento: puesto que el desenlace de la huelga general de 1988 permitió a los sindicatos satisfacer sus exigencias sin contrapartidas, el gobierno pasó desde una estrategia de asegurar el crecimiento como pre-condición de la redistribución, a otra de redistribución sin apenas condiciones.

De acuerdo con una interpretación que he repetido ad nauseam (González, 1996 y 1998), el cambio estratégico significó, de hecho, que el gobierno asumió el llamado giro social para satisfacer a los sindicatos y reponerse de la crisis de autoridad moral tras el varapalo de la huelga general, al tiempo que los propósitos reformistas (especialmente, en materia laboral) fueron remitidos a mejores tiempos. Pero ya no hubo tiempos mejores, y las medidas de reforma laboral llegaron en medio de la recesión y de la destrucción de empleo, al tiempo que se reducían los niveles de protección social (1993-94), lo que conllevó un desplazamiento de la base electoral socialista desde las clases trabajadoras que habían constituido el núcleo duro de los años ochenta (severamente castigadas por una combinación explosiva de paro, inseguridad laboral y caída de la cobertura de desempleo) hasta las clases pasivas de jubilados y amas de casa. Pero antes de seguir con la interpretación, conviene recordar los acontecimientos.

\section{LA EXPERIENCIA ESPAÑOLA (1986-1996)}

\section{Marco general}

Una de las características más sobresalientes del período que va de 1986 a 1996 es la estabilidad electoral. Por un lado, el partido socialista consigue en torno a nueve millones de votos en las cuatro elecciones generales que se celebran durante ese tiempo (en torno al $30 \%$ del censo). Por el otro, si sumamos los votos obtenidos por el PP $(5,3$ millones) y por el CDS $(1,6)$ en 1989 obtenemos una cantidad que se acerca bastante a la que obtuvo el PP en 1993 (8,2 millones), en tanto que la ganancia adicional hasta llegar a los 9,6 
RIS

REVISTA INTERNACIONAL DE SOCIOLOGIA

No 29, Mayo - Agosto, 2001

JUAN J. GONZÁLEZ RODRÍGUEZ

millones de votos en 1996 se explica, más que nada, por un nivel de movilización electoral que no se conseguía desde 1982.

A la vista de los datos agregados, cabría pensar que los cambios se limitan a la concentración y a la movilización del voto en la parte derecha del espectro político, tal como se puede deducir del estudio de la volatilidad a partir de los datos agregados. Pero una cosa es que la volatilidad así estimada haya sido muy reducida y otra que el mercado electoral se haya mantenido estable.

Pues bien, mi interpretación del proceso se apoya en dos ideas. La primera es que, tras esta aparente estabilidad, los cambios han sido de gran importancia, hasta el punto de que se pueden interpretar en términos de ciclo electoral. De hecho, es fácilmente observable una mutación o inversión del perfil del electorado socialista, de tal manera que lo que empezó siendo, a comienzos de los ochenta, un perfil más bien joven y urbano se convirtió poco a poco en un perfil envejecido y ruralizado. Se trata, por tanto, de un ciclo en el que los cambios tienen un carácter más cualitativo que cuantitativo, es decir, afectan mucho más a la composición interna del electorado socialista que al volumen de votos conseguido por este partido, lo que quiere decir dos cosas: que ha habido transferencias continuas de votos; y que las transferencias se han producido de tal manera que las salidas han sido compensadas por la entrada de nuevos votantes.

La segunda idea es que una de las mejores explicaciones de estos cambios son las políticas de bienestar que se han aplicado durante el período de referencia. En este punto, conviene tener en cuenta que se trata de un período de implantación y/o extensión del Estado de Bienestar y que dichas políticas han afectado a sectores sociales cada vez más amplios.

En su conjunto, estas políticas han supuesto una operación redistributiva sin precedentes en España (Boix, 1996), cuyo estudio recomienda tener en cuenta, al menos, los siguientes aspectos:

1. Toda operación redistributiva implica transferencia de rentas entre unos sectores y otros, lo cual supone un aumento de la presión fiscal que recae, en particular, sobre las nuevas clases medias (ibídem).

2. Las políticas redistributivas requieren algún tipo de acuerdo estratégico con sus principales beneficiarios, representados en este caso por los sindicatos, acerca de los criterios y el alcance de la redistribución.

3. Este tipo de políticas está muy condicionado por el ciclo económico y, en este caso, por la escasa duración de la fase expansiva: 1986-91.

Pues bien, cuando analizamos el período nos encontramos, de acuerdo con esta interpretación, tres fenómenos que requieren especial atención. El primero de ellos se detecta a mediados de los años ochenta, cuando ciertos sectores de clase media retiran su apoyo al partido socialista, tal como se pudo observar a partir de las elecciones generales de 1986 (y confirmar en las municipales de 1987 y 1991). Es claro que esta retirada no se puede explicar tan sólo en 
términos de pocketbook voting, pero tampoco se puede explicar al margen del hecho, bien conocido, de que es por esa época cuando en España las clases medias empiezan a pagar impuestos de acuerdo a estándares europeos ${ }^{2}$.

De hecho, la refundación del Partido Popular, en 1988, representa, más allá del cambio de liderazgo, un giro estratégico desde planteamientos y objetivos de fuertes connotaciones religiosas y morales (LODE, aborto, etc.) hacia cuestiones de carácter económico y fiscal, con el fin de atraerse a esos sectores de clase media que habían sido muy reticentes a sumarse al proyecto de "mayoría natural" de Fraga. Desde entonces el principal hilo conductor de la estrategia del PP ha sido la oferta (y consecución final, tras su llegada al gobierno) de sustanciales rebajas fiscales para las clases medias ${ }^{3}$.

En cualquier caso, es la segunda de las cuestiones enunciadas, la conveniencia de un acuerdo estratégico con los sindicatos, la que resulta crucial en la evolución de los acontecimientos. Hay que tener en cuenta que en su segunda legislatura (1986-89), el partido socialista seguía sin tener una clara oposición política y que la oposición principal se encontraba fuera del Parlamento, desde el momento en que se inicia la aproximación de los dos principales sindicatos, que habían estado divididos y enfrentados hasta entonces. Es sabido que la confrontación entre gobierno y sindicatos fue el resultado de diversos factores, pero es claro también que el principal motivo de desencuentro fue justamente la distinta manera de entender la operación redistributiva.

En este punto, había dos planteamientos: asegurar el crecimiento, en cuanto condición previa de la redistribución, y profundizar, por tanto, en las reformas $y$, en particular, en la reforma laboral; y saldar la llamada deuda social, en cuanto compensación de un largo período de autocontención de las demandas sindicales en beneficio de la consolidación democrática y de la superación de la crisis.

No voy a entrar en los detalles del conflicto que aboca a la huelga general de diciembre de 1988 (González, 1996), sino tan sólo recordar los que, a mi juicio, fueron efectos principales de la huelga. Como efecto inmediato, los sindicatos consiguieron imponer al gobierno importantes medidas redistributivas tendentes a mejorar el sistema de pensiones, la cobertura de desempieo, etc., pero sin que

\footnotetext{
${ }^{2}$ Es dudoso, en cualquier caso, que se pueda predicar una pauta clara de comportamiento electoral por parte de las nuevas clases medias, por cuanto conviven dentro de esta categoría dos mundos diferenciados en función del sector (público/privado). Véase, al respecto, la comunicación de Pablo González (1998).

${ }^{3}$ Rebajas que constituyen, dejando aparte la unión monetaria, el principal distintivo de la actual legislatura, ya sea por la vía directa del tratamiento de las rentas del capital y de la reforma del IRPF, ya por la via indirecta del modelo de financiación autonómica.
} 
RIS

REVISTA INTERNACIONAL DE SOCIOLOGIA

No 29, Mayo - Agosto, 2001

el gobierno pudiera obtener apoyo en la línea de reformas que había emprendido con el Acuerdo Económico y Social de 1984, toda vez que los sindicatos se retiraron de la mesa de negociaciones en cuanto hubieron conseguido sus objetivos $^{4}$. Como efecto mediato, se produjo un desplazamiento de los apoyos electorales desde el sector de trabajadores más cualificado y estable (insiders) hacia los trabajadores más proletarizados y más expuestos a la eventualidad y al desempleo (outsiders). La razón de esto último es doble: por un lado, la confrontación entre gobierno y sindicatos erosionó la lealtad de los insiders, que constituyen el ámbito de implantación preferente de los sindicatos; y por otro, los outsiders se vieron favorecidos por la combinación de crecimiento del empleo y de la protección al desempleo. Se llega así, a principios de los noventa, a una situación de concentración de riesgo, por cuanto la estabilidad del gobierno dependía del apoyo de sectores sociales muy vulnerables a cambios de situación económica o a desajustes en las políticas de bienestar. Así ocurre entre 1993 y 1994, cuando la combinación de recesión, desempleo, crisis del INEM, etc. se hizo socialmente explosiva con la proliferación de escándalos políticos, lo que provocó el vuelco electoral de las elecciones europeas 1994. Es claro que los factores políticos influyeron en ello, pero influyeron sobre todo como catalizadores de un proceso que se había puesto en marcha mucho antes y que culmina con la retirada de los outsiders, el sector más castigado por la crisis. La nueva situación da lugar a un cambio drástico en el perfil del electorado socialista, con un desplazamiento del centro de gravedad hacia sectores de clases pasivas: jubilados, amas de casa, etc., para quienes los beneficios de la operación redistributiva fueron mucho menos coyunturales (universalización de pensiones, sanidad, educación, etc.). De ahí el papel que han jugado como "cortafuegos electorales" en 1996, acudiendo en apoyo del PSOE hasta el punto de compensar la retirada de los outsiders.

\footnotetext{
${ }^{4}$ Como recordatorio del clima de opinión creado tras la huelga, sirvan de muestra unas declaraciones de José Barea a la revista España Económica, en abril de 1989. "No estoy de acuerdo con la revalorización de las pensiones al nivel de los salarios. Si queremos hacer una política redistributiva muy fuerte lo que hacemos es cargarnos el país (...). No digo que los sindicatos no tengan razón en algunas cosas, pero la negociación la tenían que haber planteado antes de la elaboración de los Presupuestos, que son expansivos y no soportan incluir ahora otros 500.000 millones de pts." (...). Respecto al gasto sanitario: "la cuestión no es discutir que la sanidad se universalice, sino que se ha hecho muy bruscamente y ha supuesto un fuerte aumento del gasto público. Este año ingresan más de un millón de nuevos beneficiarios, y la oferta sanitaria no se genera de golpe. Tenía que haberse hecho de otra forma".
} 


\section{Los datos}

Solo así se entienden los datos que presento a continuación, relativos a la base social del partido socialista (PSOE) y su evolución entre 1986 y 1996. El ejercicio que propongo es muy simple: voy a comparar el recuerdo de voto de 1986 y el de 1996, por cuanto dichas elecciones abren y cierran el período que, a mi juicio, debemos tomar como referencia en la discusión. En el primer caso, los datos están tomados de la macroencuesta de marzo de 1988 (CIS, 1737), que cuenta con 27 mil entrevistas. Aunque no es un estudio poselectoral, fue la primera vez que el CIS codificó la ocupación a tres dígitos, lo que nos permite operacionalizar modelos de clase homologados y reconocidos internacionalmente (el de Goldthorpe, en este caso).

Para analizar el voto del 3-M-96, he agregado los estudios 2207 (pre-electoral) y 2210 (poselectoral) del CIS, con lo que obtenemos una muestra de $10 \mathrm{mil}$ casos. Doy por supuesto que la intención manifestada en vísperas y el recuerdo manifestado poco después de las elecciones son equivalentes a efectos de identificar las preferencias electorales.

De acuerdo con mi interpretación de los hechos, los cambios en la composición social del electorado socialista han estado influidos por dos tipos de factores: uno, relativo a la clase social, y otro de carácter generacional. El primero lo vamos a analizar a partir del modelo de clase de Goldthorpe (el más utilizado en comparaciones internacionales), a la manera como vengo haciendo en anteriores estudios (González, 1996). Este modelo consiste en distinguir entre clases activas (los individuos vinculados al mercado de trabajo: en total, unos quince millones de electores a lo largo del período, incluyendo a los parados) y clases pasivas, entendiendo por tales: a) los que ya han salido del mercado de trabajo (jubilados), b) los que están al margen del mercado de trabajo (amas de casa) y c) los que todavía no han accedido al mercado de trabajo: estudiantes y buscadores de primer empleo (a los que podemos llamar jóvenes en sentido sociológico). En total, las clases pasivas representan otros quince millones de votantes, aproximadamente, de manera que el mercado electoral ha estado dividido, a lo largo del período, a partes iguales entre activos y pasivos.

La premisa básica del modelo es que las preferencias electorales están muy influidas, en el caso de los activos, por políticas fiscales y laborales, en tanto que los pasivos son más sensibles, en cambio, a las políticas de bienestar, de acuerdo con lo expuesto anteriormente.

El segundo de los factores que influye en la composición social del electorado es un factor generacional que vamos a analizar en términos de cohorte. Con tal fin vamos a comparar las cohortes decenales de 1986 con ellas mismas diez años más tarde, para lo cual podemos suponer, por ejemplo, que la cohorte entre $25 \mathrm{y}$ 34 años de 1986 es la misma que la de 35-44 de 1996. 
Este supuesto no vale, en cambio, para cada una de las combinaciones de clase y cohorte (las casillas de la tabla). Podría valer cuando se trata de categorías caracterizadas por una alta estabilidad laboral, como, por ejemplo, las clases medias de edades intermedias (podríamos suponer, en tal caso, que la mayor parte de los individuos que componen dichas categorías se mantienen a lo largo del período - me refiero, obviamente, al universo, no a la muestra). Pero no vale para las categorías internamente más inestables, como los parados, debido a la rotación (a diferencia de las clases activas propiamente dichas, el colectivo de parados se renueva casi por completo al cabo de pocos años).

Mención aparte merecen las categorías de jubilados y jóvenes, de las cuales tomamos como referencia una única cohorte (la más numerosa), cuya continuidad está limitada a lo largo del período, pues, en el caso de los jubilados, la cohorte que en 1996 tenía entre 65 y 74 años se compone de los que ya estaban jubilados (prejubilados, habría que decir) en 1986 más los que se jubilaron a lo largo de la década. Por el contrario, la cohorte de jóvenes que tenía menos de 25 años en 1986 incluye a los jóvenes de diez años más tarde (y que tenían 25-34 años en 1996, los cuales seguían sin entrar en el mercado de trabajo) más los que entraron en el mercado de trabajo a lo largo de la década.

La tabla 1 nos indica la medida en que cada una de las combinaciones de clase y cohorte se desvió, en términos porcentuales, de la media de votos obtenidos por el PSOE en 1986 (8,9 millones; $44 \%$ sobre voto válido; $30 \%$ sobre censo $^{5}$ ). En negrita aparece la zona de implantación preferente, entendiendo por tal el conjunto de celdas que se desvían 8 puntos o más por encima de la media ( $>20 \%$ de desviación). En cursiva aparece, en cambio, la zona de rechazo, entendiendo por tal las celdas que se desvían 8 puntos o más por debajo.

Desde este punto de vista, el escenario político-electoral de 1986 era un escenario típico de oposición y conflicto de intereses entre, por un lado, trabajadores manuales (especialmente no cualificados) y parados (recordemos que la mayor parte del paro se compone de trabajadores manuales, especialmente no cualificados) y, por otro, viejas y nuevas clases medias. El factor generacional estaba ligado al factor de clase, de manera que los apoyos al PSOE disminuían con la edad al tiempo que los rechazos aumentaban, pero era la clase (en su sentido estricto, como clase activa) la que marcaba la frontera entre las zonas de apoyo y de rechazo. En consecuencia, el factor generacional no tenía todavía entidad por sí mismo (obsérvese que en el marginal de columna las desviaciones

\footnotetext{
${ }^{5}$ Las desviaciones están calculadas tras la ponderación de ambas muestras (1986 y 1996) por recuerdo de voto, de manera que las medias para el conjunto de cada muestra son $44 \%$ en 1986 (43\% para las cohortes que aparecen en la tabla: $18-65)$ y $37,5 \%$ en $1996(40 \%$ para las cohortes seleccionadas: $25-75$ ).
} 
de las cohortes son muy pequeñas o nulas, a diferencia de los marginales de fila, indicativos de un escenario que puede interpretarse todavía en términos de conflicto clasista).

La tabla 2 nos indica la medida en que cada una de las combinaciones de clase y cohorte se desvió, en términos porcentuales, de la media de votos obtenidos por el PSOE en 1996 (9,3 millones; 37\% sobre voto válido; 29\% sobre censo). En relación a 1986, la zona de implantación preferente correspondiente a 1996 se ha desplazado desde los trabajadores manuales más jóvenes a los más viejos y, lo que es más significativo, a las clases pasivas de jubilados y amas de casa, dando lugar a una mutación de lo que era un perfil netamente clasista en un perfil de corte generacional. Ahora sí que el factor generacional tiene entidad propia, como lo prueban tanto los marginales de fila (con la oposición entre jubilados y jóvenes) como los de columna.

La tabla 3 presenta el balance del período, entendiendo por tal las diferencias entre las desviaciones observadas para cada una de las casillas, lo que nos permite identificar las entradas y las salidas de votos. Empezando por estas últimas, nos encontramos que las salidas más significativas (las de ocho puntos o más) están localizadas transversalmente en la cohorte que tenía menos de 25 años en 1986 y entre 25 y 34 años en 1996. Pero son especialmente significativas entre los trabajadores manuales, donde pueden observarse también salidas por encima de ocho puntos en las cohortes siguientes, de modo que las categorías de manuales no cualificados y parados arrojan en conjunto salidas por encima de los ocho puntos (véanse los marginales de fila).

Curiosamente sólo hay entradas de más de ocho puntos en una de las casillas de la tabla: las amas de casa de la última cohorte, pero, aunque más modestas, hay entradas a través de casi toda esta última cohorte. Puesto que los saldos de los marginales (tanto de columna como, sobre todo, de fila) son negativos, cabe preguntarse cómo es posible que estas entradas hayan compensado las salidas (más diversificadas y abultadas, a la vista de la tabla) hasta el punto de que el PSOE haya mantenido su nivel de apoyo electoral en términos absolutos en torno a los nueve millones.

Conviene tener en cuenta, no obstante, que el paso de los datos relativos (como los que se muestran en la tabla) a los absolutos se complica, al menos, por dos razones. La primera son las diferencias demográficas entre las categorías de la tabla (jubilados y amas de casa son, con diferencia, las dos más numerosas). La segunda, más dificil de demostrar, son las diferencias en su tasa de participación electoral: la tasa media de participación pasó del 70\% al 80\% entre 1986 y 1996, pero mi hipótesis es que jubilados y amas de casa aumentaron su participación por encima de la media.

Esto nos impide saber con precisión en qué medida las entradas de voto de la última cohorte (las necesarias para compensar las salidas) se deben a un aumento del apoyo al PSOE en términos relativos (por relación a los demás partidos, tal 
Tabla 1.

Balance electoral del PSOE según clase y cohorte (1986-96).

\begin{tabular}{|c|c|c|c|c|c|c|c|c|c|c|}
\hline $\begin{array}{l}\text { Cohorte } \\
1986\end{array}$ & $\begin{array}{c}\text { Viejas } \\
\text { clases } \\
\text { medias }\end{array}$ & $\begin{array}{l}\text { Nuevas } \\
\text { clases } \\
\text { medias }\end{array}$ & $\begin{array}{c}\text { No } \\
\text { Manual }\end{array}$ & $\begin{array}{c}\text { Manual } \\
\text { cualificado }\end{array}$ & $\begin{array}{l}\text { Manual no } \\
\text { cualificado }\end{array}$ & Parado & Jubilado & $\begin{array}{c}\text { Ama } \\
\text { de casa }\end{array}$ & Joven & Total \\
\hline$<25$ & -4 & -6 & +1 & +15 & +18 & +14 & & +9 & -7 & $=$ \\
\hline $25-34$ & -6 & -9 & -1 & +13 & +16 & +12 & & +6 & & +3 \\
\hline $35-44$ & -9 & -11 & -4 & +9 & +13 & +8 & & +3 & & +1 \\
\hline $45-54$ & -12 & -14 & -7 & +6 & +9 & +5 & & $=$ & & -2 \\
\hline $\begin{array}{l}55-64 \\
>64\end{array}$ & -11 & -14 & -7 & +6 & +10 & +6 & +6 & $=$ & & $=$ \\
\hline Total & -9 & -11 & -3 & 410 & +13 & +10 & +6 & +2 & -7 & $=$ \\
\hline
\end{tabular}

Tabla 2.

\begin{tabular}{|c|c|c|c|c|c|c|c|c|c|c|}
\hline $\begin{array}{l}\text { Cohorte } \\
1996\end{array}$ & $\begin{array}{l}\text { Viejas } \\
\text { clases } \\
\text { medias }\end{array}$ & $\begin{array}{c}\text { Nuevas } \\
\text { clases } \\
\text { medias }\end{array}$ & $\begin{array}{c}\text { No } \\
\text { Manual }\end{array}$ & $\begin{array}{c}\text { Manual } \\
\text { cualificado }\end{array}$ & $\begin{array}{l}\text { Manual no } \\
\text { cualificado }\end{array}$ & Parado & Jubilado & $\begin{array}{c}\text { Ama } \\
\text { de casa }\end{array}$ & Joven & Total \\
\hline \multicolumn{11}{|l|}{$<25$} \\
\hline $25-34$ & -12 & -19 & -9 & +1 & $=$ & -1 & & +1 & -15 & -6 \\
\hline $35-44$ & -9 & -16 & -6 & +4 & +4 & +3 & & +5 & & -2 \\
\hline $45-54$ & -11 & -18 & -8 & +3 & +2 & +1 & & +2 & & -3 \\
\hline $55-64$ & -11 & -17 & -7 & +4 & +2 & +1 & & +3 & & $=$ \\
\hline$>64$ & -5 & -12 & -1 & +10 & +9 & +8 & +10 & +10 & & +10 \\
\hline Total & -11 & -17 & -8 & +3 & +2 & +1 & +10 & +4 & -15 & $=$ \\
\hline
\end{tabular}

Tabla 3.

\begin{tabular}{|c|c|c|c|c|c|c|c|c|c|c|c|}
\hline $\begin{array}{l}\text { Cohorte } \\
1986\end{array}$ & e 1996 & $\begin{array}{c}\text { Viejas } \\
\text { clases } \\
\text { medias }\end{array}$ & $\begin{array}{c}\text { Nuevas } \\
\text { clases } \\
\text { medias }\end{array}$ & $\begin{array}{c}\text { No } \\
\text { Manual }\end{array}$ & $\begin{array}{c}\text { Manual } \\
\text { cualificado }\end{array}$ & $\begin{array}{l}\text { Manual no } \\
\text { cualificado }\end{array}$ & Parado & Jubilado & $\begin{array}{c}\text { Ama } \\
\text { de casa }\end{array}$ & Joven & Total \\
\hline$<25$ & $25-34$ & -8 & -13 & -10 & -14 & -18 & -15 & & -8 & -8 & -6 \\
\hline $25-34$ & $35-44$ & -3 & -7 & -5 & -9 & -12 & -9 & & -1 & & -5 \\
\hline $35-44$ & $45-54$ & -2 & -7 & -4 & -6 & -11 & -7 & & -1 & & -4 \\
\hline $45-54$ & $55-64$ & +1 & -3 & $=$ & -2 & -7 & -4 & & +3 & & +2 \\
\hline $55-64$ & $>64$ & +6 & +2 & +6 & +4 & -1 & +2 & +4 & +10 & & +10 \\
\hline Total & & -2 & -6 & -5 & -7 & -11 & -9 & +4 & +2 & -8 & $=$ \\
\hline
\end{tabular}


como se refleja en la tabla) y en qué medida están reforzadas por otros factores (aumento de la esperanza de vida, diferencias en las tasas de participación, etc.). Pese a ello, los datos permiten estimar un volumen de salidas/entradas de voto en torno al millón, lo que significa que, al menos, uno de cada nueve votantes del PSOE rotó como consecuencia de los cambios recogidos en la tabla (al margen quedan, por un lado, los cambios derivados de la demografía y, por otro, la volatilidad que se haya podido producir dentro de cada una de las celdas de la tabla).

En cualquier caso, hay dos conclusiones inmediatas que podemos extraer de este balance. La primera es que las principales salidas de votantes están localizadas en las cohortes más jóvenes de los trabajadores manuales, lo que refuerza la tesis de que el punto crucial en la evolución electoral del PSOE es la retirada de los outsiders (González, 1996). Es esta retirada la que da el triunfo al PP en 1996 y no la retirada de nuevas clases medias, como se dice a veces (esta última se había producido, en su mayor parte, en los años ochenta y era perfectamente visible en las elecciones municipales de $1991^{6}$ ).

La segunda conclusión es que los jubilados y, sobre todo, sus cónyuges (es decir las amas de casa de su misma cohorte) aumentaron su apoyo al PSOE a lo largo del período de referencia y que este aumento no se explica en términos de su clase de procedencia (es decir la que tenían antes de jubilarse), tal como he defendido con anterioridad (González, 1996). El citado Rodríguez Menés ha criticado esta idea alegando que trato a los jubilados como si no tuvieran clase (op cit: 105), pero yo no digo que no la tengan. Lo que digo es que ese dato no nos explica dicho aumento de apoyo y que tal crítica nos plantea una curiosa paradoja, pues es de suponer que la composición de clase de los jubilados no tiende a proletarizarse con el tiempo, sino al contrario, lo que contradice la crítica. En otras palabras, si intervinieran tan sólo intereses meramente clasistas, el apoyo de los jubilados al PSOE se hubiera reducido con el tiempo, en lugar de aumentar, lo que quiere decir que las políticas de bienestar contribuyeron a generar intereses más allá de los meramente clasistas y, por qué no decirlo, una identidad de colectivo pensionista a la que el PSOE supo apelar en su momento.

\footnotetext{
'Coincido en este punto con Rodríguez Menés cuando se refiere a "votantes independientes de izquierdas, localizados entre las clases medias no propietarias, que habrian finalmente optado por apoyar al PSOE (en 1996) en vez de castigarlo, ante la perspectiva de un triunfo del PP" (op cit, 111). Para mayor aclaración, véase la comunicación de Pablo González Alvarez (1998) que analiza el perfil del nuevo votante del PP del 96.
} 


\section{LAS ENSEÑANZAS}

\section{Recapitulación general}

Tal como adelanté, mi propósito es ofrecer una explicación del proceso electoral global sobre el supuesto de que tal explicación es necesariamente insuficiente y que necesita, por tanto, de explicaciones adicionales o complementarias. Pero antes de referirme a ellas me gustaría sentar algunas premisas. La primera es que carece de sentido cualquier razonamiento al modo estructuralista que intente poner por delante la posición de clase de los individuos. No es la clase el principio activo de la cadena causal que conduce al voto, sino la acción de los partidos y, más concretamente, las políticas (fiscales, laborales, sociales, etc.), de manera que más que de variable independiente, la posición de clase nos sirve de variable de control para medir el impacto diferencial de las políticas: las políticas fiscales pueden ser progresivas o regresivas, las políticas laborales pueden ser flexibilizadoras o estabilizadoras, y así sucesivamente. Hay, por supuesto, cuestiones de interés nacional que concitan el consenso de los partidos, pero el consenso suele romperse cuando llegamos a este otro tipo de cuestiones donde una misma política ofrece resultados distintos según la posición social de los afectados.

La segunda es que no tengo inconveniente en aceptar un modelo económico de explicación del voto (que considero, en mi caso, bienestarista, más que económico) a nivel micro, siempre y cuando vaya acompañado de la atención suficiente a las constricciones que el marco institucional y los agentes sociales imponen a la acción de los partidos en el nivel macro.

Con estas premisas, las clases son explicativas en la medida en que la acción política de los partidos dirime conflictos de interés que hacen posible la (re)elaboración de identidades de corte clasista. Pero sin que esto suponga despojar a las clases del privilegio ontológico que les conceden los modelos estructuralistas para dárselo a los partidos (como suelen hacer los modelos accionalistas con más o menos dosis de voluntarismo), sino tan sólo reconocer a los partidos el privilegio de la iniciativa, siempre y cuando no la pierdan en el curso de la acción política, como le ocurrió al PSOE hacia finales de los ochenta.

Este modelo de explicación no excluye los factores políticos, simplemente nos obliga a diferenciar dos tipos de factores o, mejor dicho, dos momentos políticos distintos: el momento de la acción de gobierno y el momento electoral. Tomemos, por ejemplo, el caso de la retirada de los outsiders del electorado socialista a la que hemos hecho reiterada mención por ser crucial en la evolución del PSOE. Parece claro que los outsiders se han retirado en mayor medida que los insiders, como es claro también que no se retiraron todos ${ }^{7}$. Podemos suponer, por tanto, que se hayan retirado aquellos cuya identidad política era más débil o, 
mejor aún, aquéllos para quien el conflicto entre identidad e intereses concretos era más insoportable ${ }^{8}$. La identidad nos serviría, en tal caso, para explicar por qué el grueso de estos sectores ha seguido fiel (o por qué algunos se conformaron con el voto de castigo en elecciones de segundo orden: europeas de 1994 o municipales y autonómicas de 1995), pero no tanto para explicar la retirada de los demás. Si la explicación estuviera, en cambio, en factores políticos de carácter general (la progresiva debilidad y pérdida de autoridad moral del PSOE, por ejemplo), la retirada hubiese sido de parecida intensidad en todos los sectores sociales (¿por qué las clases pasivas iban a ser menos sensibles a estos factores que los trabajadores manuales?).

Pues bien, si estamos de acuerdo en que la divisoria entre insiders y outsiders, en el caso español (como en el italiano), es de carácter eminentemente generacional, parece verosímil que los segundos dejaran de apoyar a un partido por el que sus padres ya no sentían afecto ni le reconocían autoridad moral (a raíz de la confrontación entre gobierno y sindicatos). Lo cual no hizo sino crear las condiciones psicosociales para que la infortunada reforma laboral de 1994 fuese percibida como una vuelta de tuerca más en el proceso de desregulación del mercado de trabajo y de precarización de los jóvenes a través de los contratos basura 9 . Por si faltaba algo, los escándalos de corrupción en la legislatura 1993-96 se encargó de lo demás.

Empero, la combinación de pérdida de empleo y desprotección social de los años 1993-94 es un eslabón crucial de la cadena causal a la hora de explicar esta retirada, a condición de que este tipo de fenómenos no se tomen como factores estructurales stricto sensu, sino más bien como fallos o fracasos en las políticas respectivas, tal como son percibidos por los afectados ${ }^{10}$. Lejos de

\footnotetext{
${ }^{7}$ De hecho no hace falta que lo hagan todos para que la transferencia de votos al PP tenga efectos políticos inmediatos: hay que tener en cuenta que desde 1993 las diferencias electorales entre los principales partidos nunca han sido superiores al millón de votos. Si, tal como se deduce de nuestros datos, el PSOE ha perdido en torno a un millón de votantes, bastaría (ceteris paribus) con que la mitad de ellos se transfiriesen al PP para dar la vuelta a los resultados de 1993.

${ }^{8}$ Cuando la identidad política y los intereses concretos entran en conflicto, sólo hay dos salidas: o los partidos adaptan su estrategia o los electores cambian sus preferencias.

${ }^{9} \mathrm{El}$ infortunio de dicha reforma no radicó tanto en su contenido (discutible en algunos puntos, pero globalmente positivo: véase, por ejemplo, Espina, 1999, o el artículo de Julio Segura "¿Hay que reformar el mercado de trabajo?", El País 13-1-97) como en su percepción social, muy influida por la confrontación imparable entre gobierno y sindicatos.

${ }^{11}$ Es poco verosímil, en términos de opinión pública, que cuando la situación económica era favorable, como lo fue a finales de los ochenta, ello se debiera en parte a la capacidad del partido en el gobierno para conducir al país por el buen camino, y que cuando las cosas se torcieron, ello se debiera tan sólo a factores exógenos (crisis internacional) o puramente económicos.
} 
quedar excluidos, los factores políticos están presentes desde el momento en que la percepción social de la situación económica y laboral es eminentemente política e inseparable del juicio sobre la acción de gobierno.

Otra cosa es cuando analizamos el segundo de los momentos: el momento de la decisión electoral en 1996. Esto plantea nuevas preguntas que requieren una correcta formulación, pues aquí la cuestión ya no es por qué el gobierno parecía que tenía las elecciones perdidas (cosa que, a juzgar por las encuestas, venía ocurriendo casi desde principios de legislatura), sino por qué estuvo a punto de ganarlas de nuevo. Dicho en otras palabras, una cosa es que el partido en el gobierno tuviera dificultades para mantener sus apoyos sociales como consecuencia de la acción de gobierno (ya fuera por ineficacia o por falta de confianza) y otra cosa distinta es que la oposición pudiera dar las elecciones por ganadas, tal como hizo el Partido Popular.

Más concretamente, la cuestión que se nos plantea al llegar a este punto ya no es por qué la retirada de los outsiders, sino por qué la retirada no fue tan amplia como se esperaba. En principio, hay dos tipos de problemas a la hora de explicar los resultados de las elecciones de 1996. El primero tiene que ver con el carácter imprevisto de los resultados. El segundo tiene que ver con los resultados mismos: ¿por qué esa dificultad del PP para ensanchar su base social cuando el adversario político (el PSOE) estaba contra las cuerdas?

El primer problema se explica, sobre todo, en términos del clima de opinión pública creado a lo largo de la última legislatura socialista (1993-96) y la manera como los sondeos contribuyeron a alimentar una dinámica típica de espiral del silencio en torno al electorado socialista ${ }^{11}$. El segundo problema tiene que ver, en cambio, con la estrategia desplegada por el PP a lo largo de aquella legislatura. De manera esquemática, podemos decir que dicha estrategia tuvo una doble característica: por un lado, en su intento de corregir los errores de las generales de 1993 y, en parte, de las municipales de 1995, el PP trató de dulcificar las aristas neoliberales de su discurso, eliminando o silenciando los temas más inquietantes de su programa bajo el reclamo de un nuevo centrismo ("Gana el centro", tal como rezaba el eslogan del Congreso celebrado por el PP a un mes de las elecciones). Por otro, desplegó la consabida operación de acoso y derribo contra Felipe González.

Pero ambos aspectos resultaron problemáticos. El primero, porque a medida que se acercaba la campaña electoral, las proclamas neoliberales fueron sustituidas por una nebulosa de declaraciones retóricas y vaguedades cuyo principal mensaje era que quien las decía daba por ganadas las elecciones y se sentía, por tanto, exonerado de explicar su programa. El segundo, porque, en su

"Lo he desarrollado por extenso en González, 1998. 
intento de acoso y derribo, el PP desencadenó una fuerte tensión política y una línea de comunicación que por su propia radicalidad y frontalidad dificultaba el paso de los votantes socialistas a "la nueva mayoría"

Peor aún, el silencio programático de quien daba por ganadas las elecciones fue ocupado por quienes se sentían autorizados para hablar en su nombre, ya fuese la banca o la patronal ${ }^{13}$, lo que fue interpretado por los adversarios y por una parte de la opinión pública como el "programa oculto" del PP. El resultado de todo ello es bien sabido: victoria al límite en términos de votos (poco más de un punto porcentual de diferencia respecto a los socialistas) y algo más holgada en términos de escaños (en virtud de las primas que la ley electoral concede a los partidos que ganan en las circunscripciones más pequeñas), pero lejos de la mayoría suficiente que Aznar había dado por supuesta durante su campaña.

Está claro que cualquier intento de explicar los resultados de unas elecciones debe, por lo pronto, contemplar separadamente estos dos planos: el momento de la acción de gobierno, en el que los electores evalúan dicha acción por referencia a su grado de acuerdo o satisfacción, y el momento de la decisión electoral propiamente dicha, donde la evaluación se hace por referencia a la oferta de la oposición. Como lo está también que ningún modelo de voto económico puede explicarlo todo, por más sofisticado y multicausal que sea: como acabamos de ver en nuestro ejemplo, el modelo puede explicarnos el porqué de la retirada de los outsiders, pero no su magnitud.

Sentadas estas premisas de carácter general, es el momento de formular algunas de las enseñanzas particulares que podemos extraer de la experiencia española.

\footnotetext{
${ }^{12}$ Conviene distinguir dos aspectos en la comunicación del PP: su contenido básico, articulado en torno a la consabida tríada "paro-corrupción-despilfarro" (lo que Bouza llama esquema analítico), y su presentación en el contexto de dicha operación, por cuanto la frontalidad y la globalidad de los ataques no hacían sino aumentar la distancia que los votantes socialistas debían recorrer para llegar al PP (véase Bouza, 1998). De tal manera que incluso los que racionalmente pudieran conectar con el mensaje del PP, se encontraban emocionalmente impedidos para dar el paso.

${ }^{13}$ Particularmente expresivo fue J.M ${ }^{\mathrm{a}}$. Cuevas, presidente de la patronal española CEOE, al emplazar al (futuro) nuevo gobierno a "replantearse el Estado de bienestar" (con recortes en desempleo, sanidad y pensiones) y hacer una nueva reforma laboral con un despido más barato. Las declaraciones fueron hechas a un mes de las elecciones (El País, 30-1-1996).
} 


\section{Sobre las bases sociales del voto}

La relación entre clase y voto sólo existe en la medida en que seamos capaces de identificar las variables intermedias que intervienen en la cadena causal. Esta cadena atraviesa diferentes planos, desde el nivel típicamente estructural (mercado de trabajo, estructura de clase, etc.) hasta la cultura política, pasando por variables de tipo político-institucional (las políticas de Bienestar, el marco de relaciones laborales, etc).

La tarea del analista consiste, por tanto, en especificar las variables que intervienen en ella, dando lugar a la formación de las preferencias políticas. En algunas ocasiones, una determinada fiscalidad puede ser decisiva para entender las preferencias de las nuevas clases medias, pero es probable que su impacto sea desigual en función del sector (público vs privado). En cualquier caso, es igualmente probable que no pueda explicarnos, por sí sola, la decisión electoral.

Nada más lejos de esta perspectiva que la idea de las clases sociales como "bases naturales de la política partidista", que (de acuerdo con la teoría de la demanda que discutimos al principio) permiten la formación de coaliciones en virtud de intereses y alineamientos predefinidos, las cuales permiten, a su vez, el acceso a la hegemonía. ¡Pero sin que los partidos puedan hacer gran cosa por modificar la secuencia! Este tipo de razonamiento sólo sirve para convalidar una de las falacias más corrientes del análisis político-electoral, según la cual el hecho de que los obreros voten conservador o las clases medias voten socialdemócrata constituye o una anomalía inexplicable o una prueba irrefutable del declive de las clases.

La simple idea de "bases naturales de la política" repugna la inteligencia del analista. No hay más bases sociales que las que resultan de la explicación concreta de las razones y los mecanismos por los que un colectivo presta apoyo a un partido. La principal implicación de todo esto es que los cambios en la estructura de clase influyen en la evolución del sistema de partidos, pero no pueden explicar por sí solos la suerte de los partidos. Con frecuencia se ha dicho que la reducción de la clase obrera condenaba a la socialdemocracia a la marginalidad, al quedarse sin base social. Lo que la experiencia demuestra, más bien, es que la suerte de la socialdemocracia depende, en lo fundamental, de su capacidad para conseguir coaliciones entre sectores sociales cada vez más diversificados y heterogéneos (Kitschelt, 1993a y 1993b), hasta el punto incluso de que la clase obrera deje de ser su núcleo duro, como ha ocurrido en el caso español.

Esto tiene dos tipos de consecuencias, al menos. Una para los partidos, cuya tarea consiste en conseguir coaliciones de sectores sociales cada vez más heterogéneos. La segunda es para los analistas, por cuanto la acción de los partidos se evalúa cada vez menos en términos de lo que representan (izquierda vs 
derecha, por ejemplo) y cada vez más en términos de lo que hacen (las políticas concretas, en la medida en que afectan al bienestar de los ciudadanos).

\section{Sobre la formación de "cleavages"}

En justa simetría con la idea anterior, las teorías de la oferta sostienen que los cleavages se forman como resultado de las estrategias partidistas, siendo, por tanto, relativamente manejables para los partidos omnivoros. Pero la evidencia disponible no se compadece bien con esta especie de optimismo estratégico. Dejando aparte los de corte étnico (acaso los más inmanejables), los cleavages pueden ser de dos tipos: los de tipo adquisitivo, que podemos resumir en términos de clase, y los de tipo adscriptivo, relativos al género y la edad o la generación.

En este punto, las objeciones son también de dos tipos. Es dudoso, en primer lugar, que la clase haya sido históricamente un ejemplo de cleavage manejable a partir del cual los partidos autoidentificados de izquierda pudiesen orientar su estrategia (no es cosa de repetir aquí el conocido argumento de Przeworsky sobre los dilemas de la socialdemocracia). Parece, más bien, que el desideratum de los partidos fuese disponer de un electorado de perfil difuso, donde la probabilidad de obtener apoyos sea parecida en los distintos sectores sociales, que permita hacer discursos planos con invocaciones más o menos retóricas a la modernización y a tópicos de ese estilo. De forma que cuando los partidos activan este tipo de cleavage de manera estratégica suele ser por razones estrictamente defensivas, a fin de afianzar el núcleo duro de su electorado en coyunturas adversas.

En segundo lugar, la formación de cleavages en torno a factores adscriptivos no suele ser resultado de estrategias intencionadas, sino efecto no querido de estrategias orientadas a regular o neutralizar el conflicto de clase. Así ocurre, por ejemplo, cuando el modelo de Estado de Bienestar promovido por la socialdemocracia sueca para conjurar la lucha de clases entra en crisis y da lugar a un conflicto de género, como consecuencia de la superposición entre la nueva división sexual del trabajo y la oposición entre sector público y privado (confiemos, como dice Esping-Andersen (1993), en que los lazos afectivos de la pareja neutralicen el nuevo potencial de conflicto).

En España, hemos visto cómo la base electoral del PSOE se desplazaba desde las clases trabajadoras a las clases pasivas de jubilados y amas de casa. La razón de este desplazamiento es doble. Primero, porque los beneficios obtenidos en el capítulo de "sanidad y seguridad social" eran menos vulnerables a los efectos del ciclo económico que los obtenidos en materia de empleo (incluyendo tanto las políticas de fomento del empleo como la protección del desempleo). Y, segundo, porque, más que el ciclo económico, lo que ponía en riesgo el nuevo status de estas clases pasivas era la eventual llegada de políticas neoliberales. De ahí 
las apelaciones del PSOE a estos sectores sociales a partir de 1993 y el riesgo consiguiente de formación de un cleavage de corte generacional, pero sin que pueda decirse que había intención de conseguir tal cosa.

En principio, el gobierno buscaba conciliar los intereses de jóvenes y de viejos, pero, en la práctica, concentró su esfuerzo en aquello que los sindicatos reclamaban con más intensidad ("sanidad y seguridad social"). Por supuesto, no era intención de nadie perjudicar la inserción laboral de los jóvenes, pero, en la práctica, un alto nivel de cotizaciones sociales (necesario para mantener la protección social) actúa como un impuesto sobre el empleo. Cuando el ciclo económico acompaña, el problema resulta indoloro. En caso contrario, una de dos: o los jóvenes quedan nuevamente aparcados o aparecen los contratos basura. De ahí el cleavage.

Cabría pensar, quizás, que la responsabilidad no fue tanto del gobierno como de los sindicatos, pero el problema es más complejo. En el esquema keynesiano clásico, las políticas sociales eran una especie de variable dependiente respecto de la capacidad de presión salarial. En virtud de las políticas de compromiso de clase, los sindicatos renunciaban a (parte de) su capacidad de presión sobre las empresas a cambio de políticas de bienestar (lo que tendía, de paso, a desplazar el conflicto desde el ámbito de la distribución al de la redistribución, a través de la mediación del Estado).

Hoy en día, ese vínculo de dependencia está diluido, desde el momento en que las clases pasivas han pasado a representar un tercio del censo electoral de las democracias avanzadas, lo que les permite actuar a modo de electorado bisagra cuya inclinación en uno u otro sentido es más que suficiente para dirimir cualquier litigio redistributivo. En tales condiciones, los pensionistas disponen de una especie de veto electoral a cualquier tentativa de corregir el sistema de redistribución en contra de sus intereses.

La formación del cleavage generacional tiene, por tanto, la siguiente secuencia. En un primer momento, interviene el marco de representación de intereses. Porque si bien la huelga general de 1988 se convoca, en términos declarativos, contra el Plan de Empleo Juvenil anunciado por el gobierno (lo que cabe interpretar como indicador de amplio consenso social a favor del colectivo juvenil), esto no implica que las decisiones que se toman a la salida de la huelga reflejen fielmente aquella motivación. De hecho, tales decisiones reflejaban mejor las posiciones de los negociadores que los intereses de los movilizados. En segundo lugar, interviene el desigual peso electoral de los colectivos afectados por el litigio y la consiguiente capacidad de veto de los pensionistas.

Queda, por último, la cuestión acerca de por qué la contraposición de intereses no da lugar, en este caso, a un conflicto explícito (más allá del realineamiento electoral por razón de la edad), cuestión que Garrido y Requena han formulado así: "si a los jóvenes el pacto [con el que se salda la huelga] no les pertenece, ni les representa, ni les resuelve ¿por qué lo toleran? Dejando al margen algunas 
respuestas violentas (...) la respuesta hay que encontrarla en el ámbito familiar" (Garrido y Requena, 1996: 55).

Más concretamente, estos autores proponen la idea de un pacto intergeneracional implícito como vía por la que las familias encajan, a nivel micro, los costes de decisiones a nivel macro. "Los costes que los jóvenes asumen en el espacio público, los ven compensados en el espacio privado. Mientras que el grueso de los recursos transferidos directamente en forma de rentas van en su mayor parte a los mayores, los menores reciben unos servicios familiares que acompañan a una prolongada formación estatal. De este modo la competencia laboral se equilibra por sexo y se desequilibra por edad" (ibídem: 56). Nuevamente, es dudoso que estemos ante un cleavage fácilmente manejable, pero lo que parece seguro es que por ahí pasan (algunas de) las nuevas bases sociales de la política democrática.

\section{Sobre la capacidad estratégica de los partidos}

El desplazamiento del electorado socialista es crucial para entender el dilema del PSOE en la fase recesiva del ciclo (1992-1996), cuando la agenda política estaba marcada por las exigencias de la convergencia europea y los imperativos de contención del gasto social. Pues dicho desplazamiento terminó llevando al PSOE a una contradicción entre su estrategia económica y el perfil de su base electoral, contradicción que, llevada a sus últimas consecuencias, parecía obligarle a elegir entre mantener las exigencias de austeridad y rigor presupuestario derivadas del programa de convergencia europea, en cuyo caso convenía recuperar apoyos sociales con los que ya no contaba (no, al menos, en la medida suficiente) o asegurar los apoyos disponibles, en cuyo caso era preferible una estrategia menos rigurosa.

De cara a unas elecciones generales, el problema consistía en que, de hacer esto último y orientarse hacia una política más expansiva, el PSOE podía competir en mejores condiciones con Izquierda Unida (IU) y ampliar su electorado por la izquierda, pero dejaba así demasiado margen de maniobra al Partido Popular (PP), para ocupar el centro. En tanto que, de hacer lo contrario, podía impedir esto último a riesgo de una mayor competencia por parte de IU. Es cierto que la agenda política de la última legislatura socialista (1993-1996) estuvo marcada por problemas políticos de extraordinaria importancia, pero el conocido argumento de que dichos problemas fueron la causa de la derrota socialista de 1996 no nos debería ocultar que su impacto fue decisivo precisamente porque el PSOE había agotado su capacidad de maniobra estratégica. 


\section{CONCLUSIONES}

A lo largo de este artículo, he intentado esbozar un modelo explicativo de la evolución electoral española entre 1986 y 1996 capaz de eludir los problemas de reduccionismo a que hice referencia en la primera parte. El modelo, que podemos caracterizar, a un tiempo, de interactivo (en cuanto al tipo de relación que establece entre los factores de oferta y demanda) y bienestarista (en cuanto al contenido de la relación), no sólo es compatible, sino que requiere de explicaciones complementarias, tales como las que he sugerido en la recapitulación. No se trata, por tanto, de ofrecer explicaciones omniabarcantes, sino de formular modelos con las debidas especificaciones de tiempo y lugar: lo que vale para el momento de la acción de gobierno puede no valer para el momento de la decisión electoral; y lo que vale para la España de los "ochenta puede no valer para los noventa. En cualquier caso, el modelo debe contemplar la conexión entre factores micro (la lógica de los electores) y factores macro (la lógica de los agentes sociales). El solapamiento de un conflicto de intereses de corte clasista y de un conflicto generacional en la segunda mitad de los ochenta constituye un ejemplo aleccionador de hasta qué punto dichas lógicas son autónomas. Del extraordinario protagonismo de los sindicatos no se deduce necesariamente que su actuación diese lugar a una redistribución a favor de los trabajadores. Puede que estos se vieran favorecidos, pero no tanto en su condición de trabajadores per se como en su condición de trabajadores jubilados o próximos a la jubilación. Otra cosa es que la redistribución a favor de los mayores estuviese enmascarada por la retórica más conveniente para quienes la promovían.

\section{REFERENCIAS BIBLIOGRÁFICAS}

BOIX, C. (1996), Partidos políticos, crecimiento e igualdad. Estrategias económicas conservadoras y socialdemócratas en la economía mundial, Alianza Universidad, Madrid.

BOUZA, F. (1998), "Democracia y comunicación política: paradojas de la libertad", ponencia presentada al VI Congreso Español de Sociología (A Coruña, septiembre de 1998).

FUSI, J.P. y PALAFOX (1997), España: 1808-1996. El Desafio de la Modernidad, Editorial Espasa, Madrid.

ESPINA, A. (1999), "El Guadiana de la concertación neocorporatista en España: de la huelga general de 1988 a los acuerdos de 1997", en F. Miguélez y C. Prieto (coord.), Las relaciones de empleo en España, Siglo XXI, pp. 23-50, Madrid.

ESPING-ANDERSEN, G. (1993), "Postindustrial Cleavage Structures: A Comparison of Evolving Patterns of Social Stratification in Germany, Sweden, and the United States", en Piven (ed.): Labor Parties in Postindustrial Societies, pp. 147-168. 
GARRIDO, L. y M. REQUENA (1996), La emancipación de los jóvenes en España, Instituto de la Juventud, Madrid.

GONZÁLEZ ALVAREZ, P. (1998), "Clase, voto y Estado de Bienestar en España (1986-1996)”, comunicación presentada a la sesión sobre "Clase, política y ciudadanía", VI Congreso Español de Sociología, Coruña, FES.

GONZÁLEZ, J.J. (1996), "Clases, ciudadanos y clases de ciudadanos. El ciclo electoral del pos-socialismo (1986-1994)", $R E I S, \mathrm{n}^{\circ} 74, \mathrm{pp} .45-76, \mathrm{CIS}$, Madrid. (Hay versión inglesa en la English Edition 1997 de la REIS, pp. 125-155).

(1998), "Política y demoscopia. Los sondeos y las elecciones generales de 1996", Empiria, Revista de metodología de ciencias sociales, $\mathrm{n}^{\circ}$ 1, pp. 181-199.

KITSCHELT, H. (1993a), The Transformation of European Social Democracy, Cambridge University Press.

(1993b), "Class Structure and Social Democratic Party Strategy", British Journal of Political Science, $\mathrm{n}^{\circ} 23$, pp. 299-337 (Hay versión castellana de próxima publicación en la revista Zona Abierta).

PARAMIO, L. (1998), "Clase y voto: intereses, identidades y preferencias", ponencia presentada a la sesión sobre "Clase, política y ciudadania", VI Congreso Español de Sociología (A Coruña, septiembre de 1998).

RODRÍGUEZ MENÉS, J. (1997), "Elecciones y hegemonía política en España”, Revista Internacional de Sociologia, $\mathrm{n}^{\circ}$ 16, pp. 83-114, CSIC, Córdoba.

TORCAL, M. y CHHIBBER (1995), "Elites, cleavages y sistema de partidos en una democracia consolidada: España (1986-1992)", REIS, nº 69, pp. 7-38, CIS, Madrid. 